\title{
Correction of Scanning Steps to Improve Accuracy in Interferometric Profilometer
}

\author{
E. Sysoev ${ }^{1}$, R. Kulikov ${ }^{1}$, I. Vykhristyuk ${ }^{1}$, Yu. Chugui ${ }^{1,2,3}$ \\ ${ }^{1}$ Technological Design Institute of Scientific Instrument Engineering, Siberian Branch \\ of the Russian Academy of Sciences (TDI SIE SB RAS), \\ 41, Russkaya str., Novosibirsk, 630058, Russia, chugui@tdisie.nsc.ru \\ ${ }^{2}$ Novosibirsk State University, 2, Pirogova str., Novosibirsk, 630090, Russia \\ ${ }^{3}$ Novosibirsk State Technical University, 20, K. Marksa pr., Novosibirsk, 630073, Russia
}

\begin{abstract}
In scanning interferometry of longitudinal shift, an uncertainty of required phase shift performance leads to a measurement error. Such uncertainty can be caused by external factors (vibrations, air turbulence in measuring area etc.) as well as inaccuracy of the scanning system. The method for calculating the phase shift between interferograms, which allows reducing the measurement error, is proposed. The results of numerical and full scale experiments are presented.
\end{abstract}

Keywords: Phase shifting interferometry, nanorelief measurement, measurement error.

\section{INTRODUCTION}

$\mathrm{C}$ URRENTLY, the technique of surface topography measuring using low-coherence interferometry methods is well known and often used to solve measurement and inspection tasks. High accuracy is achieved by determining the phase of the light scattered by different regions of the surface under inspection. During measurement the scanning of the whole interferometer or its part (e.g. mirror in the reference channel) at a predetermined pitch for receiving a set of interferograms is performed. The dependence of the light intensity from the scanning position (correlogram) for each point of surface being measured can be obtained by a set of interferograms. Using the Fourier analysis or the method of minimization of correlogram deviation allows calculating the absolute height of the surface or height difference from a selected region.

In present time a lot of attention is paid to improvement of interference measurement accuracy [1-4]. The presence of noise in interferograms and non-linearity of the scanning system leads to distortion of the correlogram functions and error in the measurement results. Random phase shifts caused by vibrations of the interferometer or turbulence of the medium, in which interfering light fields are being spread, increases the amplitude noise in correlograms and leads to an increase of the random error of the relief height measurement. Nonlinearity of the scanning system also adds uncertainty in the magnitude of the scanning steps and increases the systematic error. This is especially relevant when the difficulties with the nonlinearity of the scanning system correction (such as lack of repeatability) appear.

The aim of this work is to improve the accuracy of a nanorelief surface measurement by reducing the uncertainty of the scanning process and accounting of distortions in the correlograms. This is particularly important in order to achieve the capability limit of height resolution.

For this purpose, the authors suggested a method of calculating the scanning steps which is based on the fact that during measuring the intensity of all pixels in each interferogram is changed synchronously.

\section{STATEMENT OF THE PROBLEM}

Nanorelief surface is calculated by a set of interferograms, the number of which may vary from one to several hundred. A large number of interferograms (more than nine) is used in cases when the measured surface relief height is calculated using the Fourier analysis or the method of correlogram difference minimization. The intensity in each interferogram for each point of the measured surface depends on height $h$ of this point and the difference of optical paths $z$ in the reference and measuring arm of the interferometer. To obtain the required number of interferograms, coordinate $z$ is scanned with the predetermined step $\Delta z$. At every scanning step $k$ ( $k \in\{0,1, \ldots K-1\}$, where $K$ is the number of scanning steps), the interferogram $I_{k}$ is recorded.

In the case of interference of partially coherent wave the intensity distribution of the interferogram is described by the known function [5]:

$$
I_{k}=I_{0}\left[1+b\left(h, z_{k}\right) \cos \left(4 \pi \frac{z_{k}-h}{\bar{\lambda}}\right)\right],
$$

where $I_{0}$ is the average value of the intensity, $z_{k}=z_{0}+k \Delta z$ is the value of scanning coordinate for step $k, z_{0}$ is the initial value of the coordinate, $\bar{\lambda}$ is the effective wavelength, $b$ is the module of the complex degree of coherence. When using a light source with a Gaussian spectrum power density the module of the complex degree of coherence can be written as:

$$
b(h, z)=\exp \left(-4 \ln 2\left[\frac{z-h}{l_{c}}\right]^{2}\right),
$$

where $l_{c}$ is the coherence length of the light source. 
Even at high scanning accuracy the difference between the value $z_{k}$ and the real change $\widetilde{z}_{k}$ of the optical path can arise. This is due to the vibration or air turbulence inside the interferometer or in the measurement area.

As an example, Fig.1. shows results of simulation of correlogram in the selected point of the measured surface in the presence of random and systematic error in the phase shift between interferograms and in their absence.

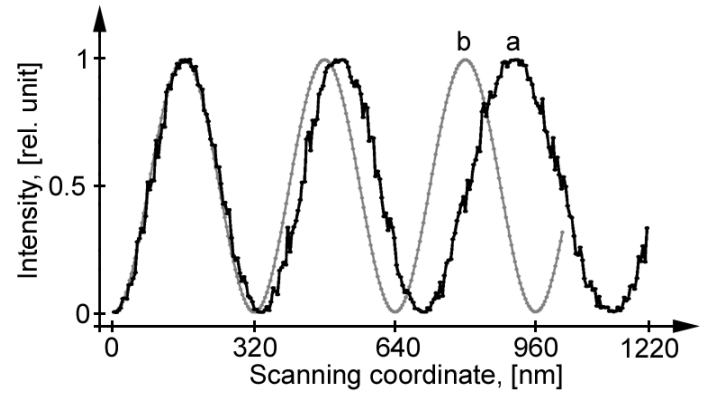

Fig.1. Correlogram: a - in the presence of phase noise and nonlinearity of the scanning system, $b$ - with regular scanning step without phase noise.

By above reasons, during scanning the main contribution to the error of the phase change is made synchronously for all points of the interferogram.

To reduce the measurement error in this paper we propose the method of calculating the scanning steps $\widetilde{z}_{k}$ by a set of interferograms and using these values under reconstruction of a surface nanorelief.

\section{SCANNING STEPS CALCULATION}

To eliminate the influence of nonuniformity of such parameters in the interferogram as background intensity, the scattering properties of the surface and contrast of the interference fringes, the normalization of the original set of interferograms is performed. For this purpose the normalization of signal amplitude in each correlogram is produced. Then, expression (1) describing the interferogram intensity is converted to the form:

$$
\hat{I}_{k}=\cos \left(4 \pi \frac{\widetilde{z}_{k}-h}{\bar{\lambda}}\right)
$$

Problem of $\widetilde{z}_{k}$ calculation is solved by finding a scanning coordinate change between arbitrary interferograms from the resulting set. To determine the shift $\Delta \widetilde{z}_{m, n}=\widetilde{z}_{m}-\widetilde{z}_{n}$ between interferograms obtained by scanning steps $m$ and $n(m, n \in\{0,1, \ldots K-1\}, m>n)$, the point cloud $p$ for all the points of the surface being measured is constructed in the $X Y$ plane by the following rule: $X$ coordinate has value of point intensity in $\hat{I}_{m}, Y$-point intensity in $\hat{I}_{n}$ :

$$
p=\left(\hat{I}_{m}, \hat{I}_{n}\right)=\left(\cos \left(4 \pi \frac{\widetilde{z}_{m}-h}{\bar{\lambda}}\right), \cos \left(4 \pi \frac{\widetilde{z}_{n}-h}{\bar{\lambda}}\right)\right)
$$

The points of $p$ lie on an ellipse centered at $(0,0)$ and rotated around the axes $X Y$ on $\pi / 4$ as shown in Fig.2. The shift between the two different interferograms in a range of $[0, \bar{\lambda} / 2]$ can be determined from parameters of the ellipse:

$$
\mathbb{\Xi}_{m, n}=\frac{\bar{\lambda} \arcsin (a b)}{4 \pi}
$$

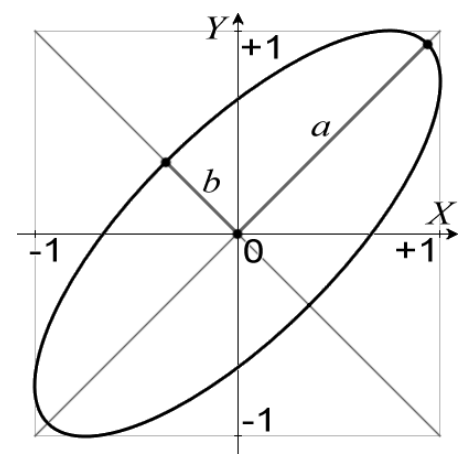

Fig.2. The locus of points $p$ for the normalized intensities of two interferograms.

The presence of amplitude noise in the interferograms leads to "thickening" of point cloud (Fig.3.a)) and small range of variation in the height of the relief $\Delta h$ leads to the fact that the intensity in the interferogram changes within less than one fringe and $p$ does not cover the whole ellipse, but only part of it (Fig.3.b)).
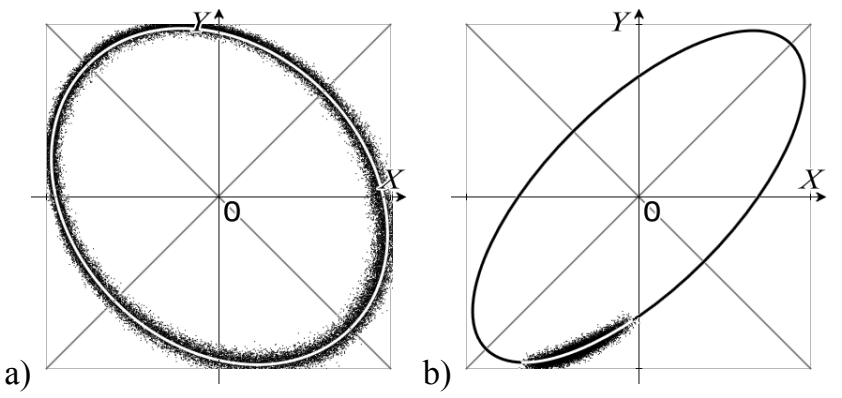

Fig.3. The point clouds in the presence of noise in the interferograms:

a) the interferograms used are from the full-scale experiment (noise level $\sim 2.5 \%$ and $\Delta h>\bar{\lambda} / 2$ ),

b) the interferograms used are from the numerical experiment (noise level $2 \%$ and $\Delta h=0,075 \cdot \bar{\lambda}$ ).

In this regard, the uncertainty in determining the parameters of an ellipse is increasing, what in accordance with expression (2) leads to an increase of $\Delta \widetilde{z}_{m, n}$ calculation error.

\section{MODELING AND NUMERICAL EXPERIMENT RESULTS}

To verify the proposed method the process of scanning of flat inclined surface was simulated. Intensity values in the interferograms were calculated in accordance with the 
expression (1). Measurement data contains $K=256$ interferograms. Interfering wave had effective wavelength $\bar{\lambda}=640 \mathrm{~nm}$ and coherent length $l_{c}=20 \mathrm{um}$.

To analyze the error $\varepsilon$ of $\widetilde{z}_{k}$ calculation, the noise level in interferograms and the slope of the scanning plane were varying, what leads to varying of the $\Delta h$. Scanning step $\Delta z$ was set to $4 \mathrm{~nm}$. Thus, in the model $z_{k}=z_{0}+k \Delta z$, $k \in\{0,1, \ldots K-1\}$. Error $\varepsilon$ was calculated as follows:

$$
\varepsilon=\sqrt{\frac{1}{K} \sum_{k=0}^{K-1}\left(\widetilde{z}_{k}-z_{k}\right)^{2}} .
$$

Fig.4. shows the dependence of $\varepsilon$ on $\Delta h$ and different noise levels in interferograms.

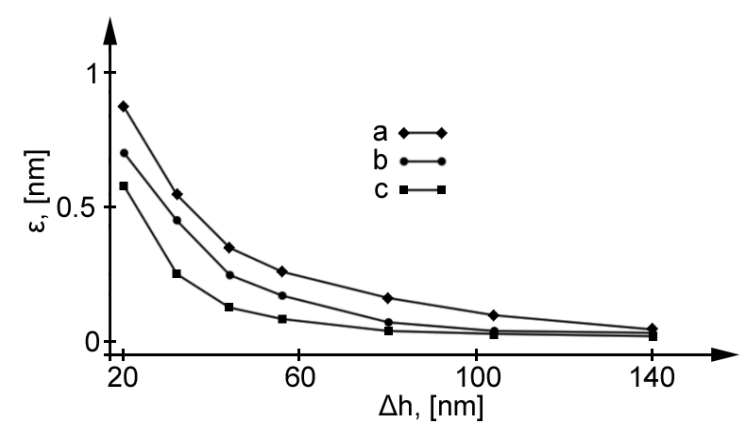

Fig.4. The dependence of $\varepsilon$ on $\Delta h$ for different noise levels: $\mathrm{a}-2 \%, \mathrm{~b}-1.5 \%, \mathrm{c}-1 \%$.

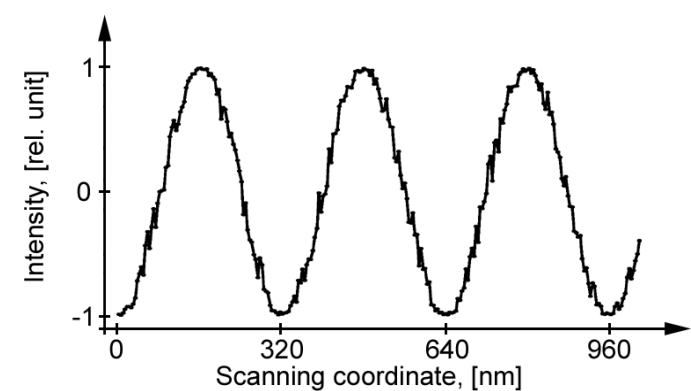

a)

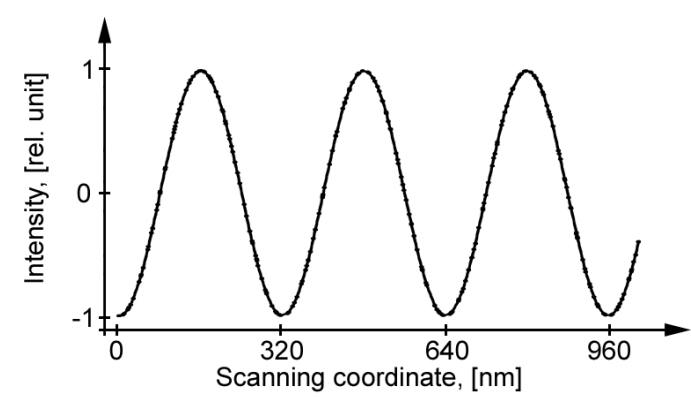

b)

Fig.5. Correlogram in the absence of amplitude noise in the interferograms, scanning step $4 \mathrm{~nm}$ and phase noise $\pm 8 \mathrm{~nm}$ : a) without accounting of the phase shift uncertainty,

b) after calculation of phase shifts by the proposed algorithm.
The graph shows that $\varepsilon$ increases with decreasing of $\Delta h$ and with increasing of amplitude noise level. However, under the condition $\Delta h>\bar{\lambda} / 4$ the influence of amplitude noise in interferograms on the $\varepsilon$ is minor.

To evaluate the effect of the proposed technique for reducing the measurement error, the simulation of measurement process by the partially scanning correlogram method [6] was performed. Amplitude noise level in the interferograms was set to $0 \%$. Scanning position uncertainty on each step was set by random value in the range of $\pm 8 \mathrm{~nm}$. This led to the appearance of the amplitude noise in the correlograms (Fig.5.a)). The level of noise does not exceed $3 \%$.

The result of correction is shown in Fig.5.b). Experiments have shown that the amplitude noise in the correlogram dropped to $0.1 \%$ which, according to the dependence given in [6], leads to reducing the measurement error from $1.5 \mathrm{~nm}$ to $0.05 \mathrm{~nm}$.

Reducing of the measurement error caused by the nonlinearity of the scanning system was estimated by simulation of the measurement of the flat inclined surface. Under condition of $10 \%$ nonlinearity the maximal error of the relief calculation was reduced from $0.67 \mathrm{~nm}$ to $0.024 \mathrm{~nm}$.

\section{FULL-SCALE EXPERIMENT RESULTS}

To verify the proposed method in full-scale experiment it was supposed to measure a flat surface and to compare a roughness of results. Measurements were carried out on an interference microscope MNP-1 [7] by the method of partially scanning correlogram. A silicon sample with nanostructure on the surface was used as a measurement object. The image of interferogram in the measurement field is shown in Fig.6. The area A inside the ellipse has roughness less than 50 picometers [8].

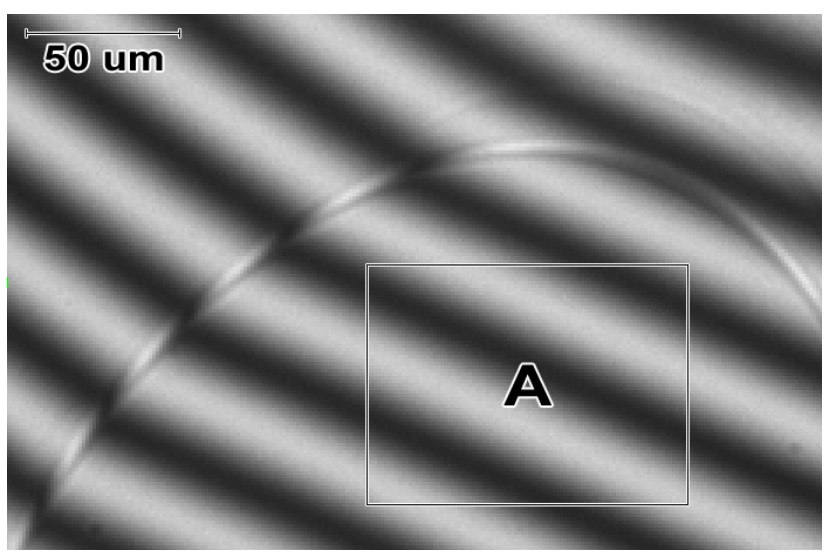

Fig.6. Image of interferogram in the measurement field.

The CCD camera Hitachi KP-F120CL was used for interferogram registration. The camera pixel size is $6.45 \mathrm{um} \times 6.45 \mathrm{um}$. The pixel size in the measurement area is equal to $0.32 \mathrm{um} \times 0.32 \mathrm{um}$. Noise ratio of registered interferograms is calculated as $\sim 2.5 \%$. 
For interferogram registration with predefined phase shift in MNP-1 the scanning process is carried out. Using a piezoelectric actuator the reference flat mirror is moved at a predetermined step $(\sim 4 \mathrm{~nm})$ along the optical axis of the interferometer reference arm. Additional phase shift that simulates the external disturbance is added to the scanning step as a well-known value, randomly selected from a range of $\pm 8 \mathrm{~nm}$.

The surface relief was reconstructed for 3 conditions: without additives to scanning step, with the additives in the range of $\pm 8 \mathrm{~nm}$, and reconstruction of surface nanorelief with additives to scanning step with a preliminary application of the proposed method.

For each reconstruction root mean square of heights of the relief $\left(\sigma_{h}\right)$ in area $\mathrm{A}$ has been calculated. The results are presented in Table 1.

Table 1. The results of calculation of $\sigma_{h}$ for reconstructed surfaces.

\begin{tabular}{|l|l|}
\hline Condition & $\boldsymbol{\sigma}_{\boldsymbol{h}},[\mathbf{n m}]$ \\
\hline without phase noise & 0.646 \\
\hline with $\pm 8 \mathrm{~nm}$ phase noise & 1.033 \\
\hline $\begin{array}{l}\text { with } \pm 8 \mathrm{~nm} \text { phase noise and with using the } \\
\text { proposed method }\end{array}$ & 0.554 \\
\hline
\end{tabular}

The improvement of measurement resolution of third reconstruction in comparison with the first one can be explained by presence of uncontrolled uncertainty of scanning steps (external vibration, air turbulence, piezoelectric actuator inaccuracy) and reducing it with using the proposed method.

\section{CONCLUSION}

The method for reducing the error of nanorelief surface measurement by the longitudinal shift interferometer, based on the calculation of the phase shifts between the interferograms is proposed.

Numerical experiments have shown that the resolution of measurements under condition of vibrations and low noise in interferograms (less than $0.1 \%$ ) can be increased up to 10 times using this method.

Full scale experiments have shown 2 times increasing of measurement resolution in presence of phase uncertainty and $2.5 \%$ noise level in interferograms.

The research results can be used for reducing measurement errors in longitudinal shift interferometers. It allows one to achieve new limit of the height resolution and to extend the scope of measurement methods based on light interference.

\section{ACKNOWLEDGMENTS}

This material is based upon work supported by the Ministry of Education and Science of the Russian Federation under Grant № 14.604.21.0086.

\section{REFERENCES}

[1] Jiang, X., Wang, K., Gao, F., Muhamedsalih, H., (2010). Fast surface measurement using wavelength scanning interferometry with compensation of environmental noise. Applied Optics, 49 (15), 29032909.

[2] Guzhov, V.I., Il'yinykh, S.P., Khaidukov, D.S., Vagizov, A.R. (2011). Eliminating phase-shift errors in interferometry. Optoelectronics, Instrumentation and Data Processing, 47 (1), 67-80.

[3] Davila, A., Huntley, J.M., Pallikarakis, C., Ruiz, P.D., Coupland, J.M. (2012). Simultaneous wavenumber measurement and coherence detection using temporal phase unwrapping. Applied Optics, 51, 558-567.

[4] Li, J., Wang, Yu., Meng, X., Yang, X., Wang, Q. (2013). An evaluation method for phase shift extraction algorithms in generalized phase-shifting interferometry. Journal of Optics, 15 (10), 5408.

[5] Fleischer, M., Windecker, R., Tiziani, H.J. (2001). Theoretical limits of scanning white-light interferometry signal evaluation algorithms. Applied Optics, 40 (17), 2815-2820.

[6] Sysoev, E.V. (2007). White-light interferometer with partial correlogram scanning. Optoelectronics, Instrumentation and Data Processing, 43 (1), 83-89.

[7] Sysoev, E.V., Vykhristuk, I.A., Kulikov, R.V., Potashnikov, A.K., Razum, V.A., Stepnov, L.M. (2010). Interference microscope-profilometer. Optoelectronics, Instrumentation and Data Processing, 46 (2), 198-205.

[8] Sheglov, D.V., Kosolobov, S.S., Fedina, L.I., Rodyakina, E.E., Gutakovskii, A.K., Sitnikov, S.V., Kozhukhov, A.S., Zagarskikh, S.A., Kopytov, V.V., Evgrafov, V.I., Shuvalov, G.V., Matveichuk, V.F., Latyshev, A.V. (2013). High-precision nanoscale length measurement. Nanotechnologies in Russia, 8 (7-8), 518-531.

Received October 3, 2014. Accepted January 24, 2015. 